\title{
Evaluation of Total Antioxidant Activity of Different Floral Sources of Honeys Using Crosslinked Hydrogels
}

\author{
Yuhong Zheng ${ }^{1,2,3}$, Mengyao $\mathrm{Wu}^{4}$, Fugui Han ${ }^{2,3}$, Dihua $\mathrm{Wu}^{1,4^{*}}$ and $\mathrm{Li} \mathrm{Fu}^{* 1,4}$ \\ ${ }^{1}$ Key Laboratory of Bee Products for Quality and Safety Control, Ministry of Agriculture, Institute of \\ Apicultural Research, Chinese Academy of Agricultural Sciences Beijing 100093, PR China \\ ${ }^{2}$ Institute of Botany, Jiangsu Province and Chinese Academy of Sciences (Nanjing Botanical Garden \\ Mem. Sun Yat-Sen), Nanjing, PR China \\ ${ }^{3}$ The Jiangsu Provincial Platform for Conservation and Utilization of Agricultural \\ Germplasm, Nanjing, PR China \\ ${ }^{4}$ College of Materials and Environmental Engineering, Hangzhou Dianzi University, Hangzhou \\ 310018, PR China \\ *E-mail: fuli@hdu.edu.cn,wudihua@hud.edu.cn
}

doi: $10.20964 / 2019.02 .71$

Received: 19 October 2018 / Accepted: 11 December 2018 / Published: 5 January 2019

\begin{abstract}
Antioxidants in traditional food can protect organisms from free radicals and prevent the occurrence and development of cancer, cardiovascular and cerebrovascular diseases and diabetes mellitus. The accurate and quick evaluation of total antioxidant activity (TAC) in food samples remains a challenge. In this work, a zinc ion-crosslinked chitosan hydrogel was prepared to evaluate the TAC of honey from different floral sources. After optimization, locust honey, linden honey, jujube honey, wattle honey, citrus honey and Chinese milk vetch honey were used as experimental samples. The results indicate that Chinese milk vetch honey exhibits the highest TAC of the honeys from the studied floral sources.
\end{abstract}

Keywords: Electrochemistry; Total antioxidant activity; Hydrogel; Honey; Metal ions

\section{$\underline{\text { FULL TEXT }}$}

(C) 2019 The Authors. Published by ESG (www.electrochemsci.org). This article is an open access article distributed under the terms and conditions of the Creative Commons Attribution license (http://creativecommons.org/licenses/by/4.0/). 